\title{
La participation des personnes utilisatrices aux exercices de planification et d'organisation des services de santé mentale au Québec
}

\author{
Participação dos usuários no planejamento \\ e organização dos serviços de saúde mental no Quebec
}

Michèle Clément ${ }^{1}$

Lourdes Rodriguez del Barrio ${ }^{1}$

Jean Gagné ${ }^{3}$

Annie Lévesque ${ }^{1}$

\footnotetext{
${ }^{1}$ Centre de santé et des services sociaux de la Vieille-Capitale. Centre affilié universitaire 880, Rue Père-Marquette. GIS-2A4 Québec Montréal. michele.clement@csssvc.qc.ca ${ }^{2}$ Université de Montréal.

${ }^{3}$ TéluQ.
}

Résumé Cet article documente la manière dont s'est renouvelé et s'est transformé au fil du temps le projet de faire participer les usagers aux exercices de planification et d'organisation des services de santé mentale au Québec (Canada). Pour ce faire, les auteurs reviennent sur l'ensemble des documents ministériels qui ont traité de cette question et dégage, pour les principaux moment-clés, les principales modalités de participation des usagers. Mots-clés Participation publique, Usagers des services de santé mentale, Gouvernance
Resumo Este artigo documenta a forma como se renovou e se transformou, ao longo do tempo, o projeto de participação dos usuários no planejamento e organização dos serviços de Saúde Mental no Quebec (Canadá). Para isso, os autores reavaliaram todo o conjunto de documentos ministeriais que trataram desta questão e retiraram, para os momentos-chaves, as principais modalidades de participação dos usuários.

Palavras-chave Participação pública, Usuários de serviços de saúde mental, Governança 
À l'instar du Canada, au Québec la participation publique à la gouvernance est une des valeurs phares des services de santé et des services sociaux. Par contre, dans le champ de la santé mentale, ce n'est que depuis les vingt-cinq dernières années qu'on a vu se mettre en place la participation des personnes utilisatrices de services (usagers) à la gouvernance du système de soins. Dans cet article, nous proposons de documenter comment s'est renouvelé et s'est transformé au fil du temps ce projet de faire participer les usagers aux exercices de planification et d'organisation des services de santé mentale.

Pour ce faire, une définition sommaire de la "participation publique" sera d'abord présentée. Ensuite, l'article sera consacré à l'analyse des principaux documents ministériels traitant de la participation des usagers à la gouvernance du système public de services en santé mentale ainsi qu'aux modalités de cette participation. La période couverte par cet exercice s'étend de 1989, année où est parue la première Politique de santé mentale au Québec ${ }^{1}$, jusqu'à aujourd'hui. L'article se termine sur un certain nombre d'enjeux en lien avec la participation publique des usagers aux exercices de planification et d'organisation des services de santé mentale.

Précisons que si nous empruntons la perspective des documents ministériels pour dégager la manière dont s'est pensée la participation des usagers à la gouvernance du réseau public de services de santé mentale cela ne signifie pas que le projet de faire participer ces personnes s'est construit en autarcie et sans lien avec la société civile. Dans le champ de la santé mentale, les usagers et les groupes communautaires autonomes (mouvement visant la transformation sociale par l'initiative et la participation citoyenne) se distinguent par leur volonté et leur détermination à participer à la gouvernance du système de santé.

\section{La participation publique}

Dans les sociétés démocratiques, la participation publique est vue comme une condition au développement social; elle permet de participer, à divers degrés, aux processus décisionnels liés aux politiques publiques afin de les améliorer. On peut définir la participation publique comme étant: l'État qui invite des citoyennes et citoyens à participer, à divers degrés, à la prise de décision, à titre d'acteurs de la société ancrés dans des pratiques (professionnelles, politiques ou communautaires) et des contextes sociaux ou politiques spécifiques, ou à titre d'individus représentatifs de la popula- tion ${ }^{2}$. Ainsi, lorsque les acteurs du réseau public de services en santé mentale invitent les usagers à participer aux exercices de planification et d'organisation des services, ils contribuent à démocratiser ce système $e^{2-4}$. Rappelons qu'une distinction importante doit être faite entre la "participation citoyenne" (initiée par la société civile) et la 'participation publique' (initiée et encadrée par l'État démocratique $)^{2}$. La participation publique est un mouvement initié par les organisations vers leurs clientèles, par les gouvernants vers les gouvernés, et non l'inverse.

\section{La participation des usagers à la gouvernance des services publics de santé mentale: \\ Analyse des documents ministériels}

Trois moments-clés nous semblent avoir structuré les efforts consentis par l'État pour penser la participation des usagers dans le champ plus spécifique de la santé mentale: 1) les travaux autour de la Politique de santé mentale de 1989 (1987 à 1997),2) les travaux autour de la réforme du réseau public de services en santé mentale (1997 à 2004) et 3) les travaux autour de la réforme de l'ensemble du réseau public de services de santé au Québec (2004 à aujourd'hui). C'est au cours de cette dernière période que sera adopté le second plan d'action en santé mentale.

\section{De 1987 à 1997: les travaux autour de la Politique de santé mentale du Québec}

L'idée d'impliquer les usagers dans les lieux où l'on discute de la planification et de l'organisation des services de santé mentale fera une avancée importante en 1987, lorsque paraît le rapport préparatoire à la première politique de santé mentale du Québec: Pour un partenariat élargi: projet de politique de santé mentale pour le Québec (Rapport Harnois $)^{5}$. Quelques usagers sont consultés dans la démarche de production de ce rapport, car on considère que la façon la plus pertinente d'examiner la situation qui prévaut en santé mentale est d'évoquer directement les propos de ceux et de celles qui y sont engagés. D’ailleurs, le soustitre du premier chapitre, "Je suis une personne, pas une maladie" reprend les propos d'un utilisateur, lesquels deviendront un slogan emblématique dans le champ de la santé mentale. Toutefois, dans cette sous-section du rapport, les propos des utilisateurs sont, au final, très peu développés.

Le rapport contient aussi une proposition de "cadre de référence" en vue de l'élaboration pro- 
chaine d'une Politique de santé mentale pour le Québec. Un des principes généraux du "cadre de référence" insiste sur l'importance d'accorder davantage de place aux usagers: "Le respect de la personne exige $[\ldots]$ de miser sur son potentiel, d'encourager sa participation et de favoriser la contribution des acteurs et des milieux qui lui sont significatifs"'. On insiste également sur l'importance de développer un "partenariat" impliquant une mobilisation concertée des différents acteurs (usagers, proches, intervenants du secteur public et communautaire) $)^{5}$. L'idée d'entendre le point de vue de l'usager et d'établir avec lui un partenariat semble donc présente, notamment en ce qui concerne la question du traitement, mais rien n'est avancé, ni même pensé pour concrétiser cette idée.

Deux ans après la publication du rapport préparatoire, la première Politique de santé mentale ${ }^{1}$ du Québec paraît. Cette dernière met de l'avant certaines orientations dont les principales sont: assurer la primauté de la personne, accroître la qualité des services, atteindre une équité régionale dans les services, rechercher les solutions dans le milieu de vie des usagers et consolider le partenariat en reconnaissant le potentiel de chacun ${ }^{6}$. La Politique de santé mentale traite plus directement de la question de la participation des usagers. On y soutient en effet qu'assurer la primauté de la personne [...] c'est également miser sur sa capacité, sur son point de vue, favoriser sa participation et celle de ses proches. Cette orientation suppose enfin sa participation dans les décisions qui la concernent"'.

Sur le plan clinique, la Politique de santé mentale valorisait la mise en place de "plan de services individualisés" (PSI) pour permettre aux intervenants d'avoir une lecture commune de la situation de l'usager, mais aussi de planifier les services et de coordonner les interventions réalisées pour la personne dans le rétablissement de son équilibre. Sous forme écrite, donc un véritable engagement, le PSI était réalisé en équipe et si possible, en présence de l'usager qui est invité à exprimer ses besoins, ses attentes et son projet de vie. Ainsi, en valorisant l'implication de l'usager, la Politique de santé mentale a certainement contribué à changer le regard posé sur ce dernier. Toutefois, sa participation se limite aux décisions cliniques et son concours à la gouvernance du système de santé demeure largement impensé.

La Politique de santé mentale de 1989 s'est aussi traduite par une importante restructuration des services de santé mentale dans toutes les régions du Québec et par l'ouverture du milieu institutionnel à travailler en partenariat avec les groupes des milieux communautaires. Chaque région devait produire son Plan régional d'organisation des services (PROS) en confiant cette responsabilité à un comité tripartite composé d'un "tiers institutionnel", d'un "tiers communautaire" et d'un "tiers autres acteurs du milieu". C'est dans ce contexte que les ressources alternatives en santé mentale (qui se différencient du modèle biomédical et s'ancrent dans un mouvement social alternatif) ainsi que les groupes de défense de droits en santé mentale du Québec (créés lors de l'adoption de la Politique de santé mentale de 1989 et dont la gestion a été confiée au milieu communautaire) seront officiellement impliqués en tant que représentants du "tiers communautaire". Toutefois, aucune place n'est encore spécifiquement attitrée aux usagers puisqu'ils agissent plutôt en tant que représentants de l'un ou l'autre des groupes communautaires impliqués dans les travaux des comités tripartites.

En 1994, le CSMQ (Comité de la santé mentale du Québec), organisme-conseil du MSSS (ministère de la Santé et des Services sociaux), publie ses Recommandations pour enrichir la Politique de santé mentale de $1989^{7}$. Le CSMQ désapprouve le fait que les usagers et leurs proches ne soient pas représentés dans les comités tripartites: "La présence des usagers et de leurs proches au sein de ces comités paraît aujourd'hui incontournable. Ce sont les premiers concernés par les résultats de la démarche de planification et le suivi [du plan de services individualisé]. Ils doivent donc être en mesure de participer activement à l'élaboration des plans régionaux d'organisation des services...", de leur implantation à leur révision$^{7}$. Le CSMQ affirme aussi que "Les personnes qui en expriment le besoin devraient également pouvoir bénéficier d'un soutien préparatoire aux réunions afin de maximiser leur participation. La nature de ce soutien devrait [toutefois] être définie en collaboration avec elles"7. En 1995 le CSMQ produit un rapport d'activités (1989-1994) intitulé Vers une politique de santé mentale de deuxième génération ${ }^{8}$. L’idée de la participation des usagers à la gouvernance du système de santé est à nouveau reprise, mais cette fois on ne la qualifie plus d' "incontournable" comme c'était le cas dans les Recommandations pour enrichir la Politique de santé mentale de $1989^{7}$, mais plutôt d' "essentielle" et la possibilité de soutenir les personnes qui souhaitent s'impliquer dans de telles activités est de nouveau soulevée: “... il est essentiel que la personne puisse participer à l'orientation et à la planification des services et 
des activités qui la concernent, et qu'elle soit aidée dans ce sens si elle en manifeste le besoin"s. Une brèche est ici ouverte. La participation des usagers ne doit pas se limiter aux décisions cliniques, mais doit également se déployer dans les exercices visant à planifier et à organiser les services de santé mentale.

Entre 1987 et 1995, on a donc vu apparaitre et évoluer l'idée de la participation des usagers. D'abord, pensée en lien avec les décisions cliniques qui les concernent pour évoluer progressivement jusqu'à la reconnaissance qu'ils doivent aussi être impliqués et soutenus dans les exercices de planification et d'organisation des services de santé mentale. Dans les faits, cependant, bien plus que les usagers eux-mêmes ce sont davantage les groupes issus des milieux communautaires qui sont impliqués dans ce type d'exercice quoique cela ne soit pas mutuellement exclusif. Cette situation s'explique possiblement par le fait que, tant au Québec qu'au Canada, les usagers vivant avec un problème de santé mentale ne se sont constitués en tant que groupes distincts que très récemment (surtout depuis 2005).

En 1997, au terme de ce que nous avons défini comme étant la première période structurante de la participation des usagers, le MSSS publie son Bilan d'implantation de la politique de santé mentale ${ }^{9}$. Pour l'essentiel, le bilan reprend les conclusions publiées un an plus tôt par le Vérificateur général du Québec (personne qui exerce un contrôle parlementaire des fonds et autres biens publics et qui rédige ses observations et recommandations dans un rapport) dans son examen du système de santé mentale. Celui-ci affirmait que le Québec était aux prises avec un important surplus de lits en psychiatrie et une insuffisance de services dans la communauté (orientation centrale dans la Politique de santé mentale de 1989). Une recommandation est alors faite au MSSS "d'assurer la transformation de l'organisation des services préconisée par la Politique de santé mentale" ${ }^{\prime 10}$. Dès lors, le ministère s'engagera dans une importante offensive afin de répondre rapidement à ce qui était préconisé par la Politique de santé mentale.

Lors du Forum consultatif organisé aux fins de la production du Bilan d'implantation de la politique de santé mentale [de 1989] et auquel participent des usagers on retiendra ceci:

... la personne n'est pratiquement jamais consultée lors de l'élaboration du PSI [plan de services individualisés] qui lui est dédié, [...] elle demeure éloignée des décisions qui la concernent. Situation qui, selon les dires des usagers et usagères, irait à l'encontre même du principe de primauté de la personne?.

Même sur le plan clinique, là où l'idée de la participation des usagers semble la plus acquise, il reste beaucoup à faire. Ainsi, bien qu'entre 1987 et 1997, l'idée d'impliquer les usagers dans la gouvernance du système de santé a passablement évoluée, peu de choses ont été faites en ce sens. Les groupes communautaires et l'ensemble des acteurs ayant participé aux Forums consultatifs arrivent aussi à cette conclusion et considèrent qu'il faut ouvrir le tripartisme à la participation des usagers et à celle de leurs proches9. Par contre, le MSSS a franchi une étape de plus dans son projet de faire participer les usagers puisque c'est en tant que groupe spécifique que les usagers ont participé au Forum consultatif. De plus, le Bilan recommande d'octroyer ... une aide financière pour favoriser la participation des milieux communautaires, des usagers et de leurs proches. ${ }^{9}$ Cette fois on ne se contente pas d'énoncer qu'il faut favoriser la participation, mais on précise enfin un moyen pour le faire: l'aide financière!

Dans les années qui vont suivre, le MSSS procédera à toute une série de travaux en vue de réaliser la première grande réforme des services de santé mentale au Québec depuis, bien sûr, le mouvement de désinstitutionnalisation amorcé au début des années 60 .

\section{La première réforme des services de santé mentale: 1997 à 2004}

À l'automne 1997, le MSSS amorce une vaste consultation en vue de dégager les orientations à prendre pour transformer les services de santé mentale rendue nécessaire par le Bilan d'implantation de la Politique de santé mentale et par les conclusions du Vérificateur Général du Québec dans son rapport de 1996. Toutes les personnes et les groupes concernés par la santé mentale seront conviés à cette consultation. Suite à cette démarche, le ministère produit un texte intitulé Orientations pour la transformation des services de santé mentale: Document de consultation ${ }^{11}$. On retrouve dans ce dernier, un ensemble de dispositions considérées comme des réponses aux problèmes soulevés par le rapport du Vérificateur général et permettant “ ... d'assurer la transformation de l'organisation des services préconisée par la Politique de santé mentale"11.

Plusieurs comités d'usagers et de bénéficiaires participent à cette consultation. Le document préparatoire à cette dernière aborde peu ou pas la question de la participation des usagers aux 
exercices de planification et d'organisation des services à deux exceptions près. Dans l'une des sections réservées à la discussion sur les services à développer dans la communauté, on réaffirme l'exigence d'impliquer l'usager dans les décisions qui le concernent. Puis, plus inhabituel, le document ouvre pour la toute première fois sur la possibilité que l'usager et ses proches puissent prendre part, en relation avec le CSMQ, à la définition des critères de qualité des services.

Ce comité, en relation avec les usagers et les usagères, les proches, les intervenants, les intervenantes, les chercheurs et les chercheuses, sera donc appelé à soutenir et à stimuler les efforts concertés en vue de l'établissement de critères de qualité11.

Un an plus tard, le MSSS va adopter un Plan d'action pour la transformation des services de santé mentale ${ }^{12}$. Pour l'essentiel, celui-ci reprend l'ensemble des dispositions mises de l'avant à l'automne 1997 dans le document de consultation ${ }^{11}$, mais cette fois avec beaucoup plus de conviction et de fermeté. Le Plan d'action reprend la question de la participation des usagers, mais autrement qu'on ne l'avait fait jusqu'à présent. La participation des usagers n'y apparait plus tant comme une fin en soi que subordonnée à l' "appropriation du pouvoir" qui est retenu comme principe directeur des changements proposés.

Cette réappropriation du pouvoir se traduit par l'exercice d'un choix libre et éclairé au moment de prendre des décisions à des étapes cruciales de sa vie. L'application de ce principe amène les intervenants et les intervenantes à respecter la personne, à l'informer, à lui offrir des recours adaptés à sa situation et, enfin, à miser sur son potentiel. Pour respecter son libre arbitre, il peut s'avérer nécessaire de lui offrir des choix thérapeutiques répondant à ses besoins.

L'appropriation du pouvoir comme démarche collective se traduit par la participation de la personne à la vie associative, tel qu'elle s'est développée dans les organismes communautaires en santé mentale. L'usager ou l'usagère sont consultés sur les modalités d'organisation des services de santé mentale, que ce soit en établissement ou en milieu communautaire. La défense des droits des usagères et des usagers constitue d'ailleurs un des fondements de cette démarche collective d'appropriation $d u$ pouvoir ${ }^{12}$.

Avec le Plan d'action, la participation devient donc une dimension de l'appropriation du pouvoir qui se déploie sur le plan personnel (clinique) ou dans une démarche collective. On reconnait que la personne est en droit de prendre davantage de place dans ses interactions avec l'in- tervenant. Mais surtout, ce qu'introduit la notion d' "appropriation de pouvoir", c'est la transformation de notre manière de concevoir, de comprendre et d'agir dans le domaine de la santé mentale ${ }^{13}$.

Les mots utilisés dans ce Plan d'action suggèrent toutefois un certain recul en ce qui concerne la participation de l'usager aux exercices de planification et d'organisation des services de santé mentale. Il ne s'agit plus tant de prendre part aux décisions comme le suggérait le Bilan d'implantation de la politique de santé mentale en revendiquant la place de l'usager au sein des comités tripartites que d'être consulté: "L'usager ou l'usagère sont consultés sur les modalités d'organisation des services de santé mentale..."9. En d'autres mots, si le Plan d'action vient consolider la participation de l'usager sur le plan clinique, il devient plus évasif et discret quant à son implication dans les exercices de planification et d'organisation des services. Nous en sommes plus au qualificatif d' "incontournable" ou d' "essentielle" tel qu'on l'avait déjà qualifiée.

Ce recul sera compensé par l'épreuve des faits. Pour accompagner la transformation des services de santé mentale, le MSSS se dote d'un Groupe d'appui à la transformation des services de santé mentale. Deux usagers de services de santé mentale y seront intégrés (Denise Blais et Francine Dorion). Pour la première fois, des usagers vont jouer un rôle important dans l'ensemble des étapes liées à la transformation des services et ils se voient reconnus comme "experts" [statut qu'ils refuseront au profit de celui de représentants des usagers]. Les membres ne représentent pas des groupes d'intérêts; ils se sont engagés à mettre à profit leur savoir pour faire des analyses articulées de la situation et produire des recommandations pour le Ministère ${ }^{14}$.

Pour assurer la représentation des usagers au sein du Groupe d'appui, le MSSS a confié le recrutement des usagers à l'Association des groupes d'intervention et de défense des droits en santé mentale du Québec (AGIDD-SMQ). Pour la première fois, le ministère s'est tourné vers une association provinciale plutôt que de s'adresser aux comités de bénéficiaires des établissements de santé mentale ou aux associations regroupant des personnes autour d'un diagnostic précis.

Rapidement, le Groupe d'appui s'engage à faire le point sur l'état de réalisation du Plan d'action pour la transformation des services de santé mentale $e^{12}$. Pour ce faire, il entame une importante tournée provinciale auprès de 26 organismes nationaux intéressés par les orientations en santé 
mentale. À la demande des représentants des usagers au Groupe d'appui, des consultations auprès des usagers et des familles seront ajoutées à l'agenda des consultations.

Au terme de cette tournée, un bilan est rédigé par les deux représentants des usagers au Groupe d'appui afin de faire ressortir les points saillants des consultations faites auprès des personnes utilisatrices de services. Le bilan a par la suite été retourné dans les différentes régions du Québec pour que les usagers puissent en discuter. Une seconde série de rencontres est alors amorcée dans chaque région du Québec pour se terminer par une rencontre nationale des usagers. C'est à ce moment que seront élus, démocratiquement, les quatre usagers qui participeront au Forum national sur la santé mentale en septembre 2000.

Deux objectifs sont poursuivis par le Forum national sur la santé mentale: 1) partager une vue d'ensemble de l'implantation du Plan d'action de 1998 et s'entendre sur les enjeux et conditions permettant d'achever la transformation des services; 2) culminer l'engagement des personnes à réaliser un plan de travail conjoint pour effectuer les changements attendus ${ }^{15}$. Les activités du Forum comprenaient des séances de travail préparatoire avec les personnes représentant les clientèles (usagers et familles) et le Forum proprement dit.

Aux dires de plusieurs personnes qui y ont été impliquées, la tenue du Forum national de septembre 2000 aura été un moment charnière au Québec en matière de participation publique des usagers et en matière de partenariat dans le secteur de la santé mentale.

La participation des personnes utilisatrices de services est un résultat important de ce partenariat qui apparaît comme une condition nécessaire à la transformation des services. Désormais, leur implication à titre de partenaires revêt un caractère incontournable à tous les niveaux ${ }^{15}$.

La participation des usagers reprend son rang de conditions nécessaires et ce, 'à tous les niveaux'. Cette prise de position se traduira concrètement dans les mesures que le MSSS mettra de l'avant en 2001, dans son document Accentuer la transformation des services de santé mentale ${ }^{15}$. On y mentionne en effet que pour la mise en place des réseaux locaux de services il faudra ... des modalités de concertation qui soutiennent la prise de décisions et qui impliquent la participation d'au moins deux personnes utilisatrices de services, des partenaires du réseau de la santé et des services sociaux, incluant les familles et les proches, ainsi que des partenaires d'autres secteurs contribuant aux besoins des personnes ayant des troubles men- taux graves $^{15}$. En ce qui a trait plus spécifiquement aux responsabilités des acteurs, il est dit que: [...] Les partenaires en santé mentale sont ainsi appelés à favoriser la participation des personnes utilisatrices de services au regard du mandat qu'ils assument, tout en offrant des conditions d'exercice adaptées à leurs capacités ${ }^{15}$.

Les travaux menés par le Groupe d'appui à la transformation des services en santé mentale auront été décisifs au regard de la participation publique: ils coïncident avec la première vraie consultation auprès d'usagers en santé mentale en tant qu' "usagers", ils élèvent la participation des usagers au rang de "condition nécessaire" et non négociable de la transformation des services et ils favorisent le développement des premières structures régionales d'organisation de la participation des usagers au regard de la gouvernance $\mathrm{du}$ système de soins de santé mentale ${ }^{16}$.

On notera cependant que malgré les efforts fournis pour achever la transformation des services de santé mentale engagés depuis le Plan d'action de 1998, le Vérificateur général du Québec soumet en 2003 un second rapport accablant. Le rapport réitère le fait que les services de santé mentale ne répondent toujours pas aux besoins de la population et que "plusieurs volets de la Politique de santé mentale de 1989 n'ont jamais été appliqués"17. Plus important encore, le rapport insiste sur le fait qu'il est impossible de tracer un bilan sur les services de santé mentale parce que peu de cibles et d'indicateurs de transformation ont été déterminés et qu'aucun système de suivi n'a été déployé. On regrette aussi l'absence d'orientations pour soutenir les services offerts aux personnes qui présentent un trouble modéré de santé mentale, la Politique de santé mentale mettant surtout l'accent sur les personnes souffrant d'un trouble grave $^{18}$. Encore inachevée, on renoncera finalement à cette réforme des services pour s'engager autrement dans une nouvelle réforme.

\section{La seconde réforme des services de santé mentale: de 2004 à aujourd'hui}

En 2004, le gouvernement du Québec passe aux mains des libéraux et ce changement se traduira par un important recul dans le champ de la santé mentale. Le CSMQ, qui se montrait progressiste et ouvertement engagé dans la cause des usagers, sera aboli et le nouveau gouvernement fera table rase des politiques et orientations mises de l'avant par le gouvernement précédent, compromettant ainsi plusieurs acquis en lien avec la participation des usagers. 
Le gouvernement libéral entreprend une réforme globale du système de santé [projet de loi no.83 $]^{19}$ avec comme orientations de fonds l'accessibilité, la coordination et la continuité des soins de première ligne, la responsabilité populationnelle et la hiérarchisation des services [réseau local de services]. Alors qu'historiquement, le champ de la santé mentale a fonctionné "en silo" et qu'il a été fortement associé aux services psychiatriques et spécialisés, la reforme entreprise par le gouvernement libéral viendra redéfinir la santé mentale comme un secteur clé des soins de première ligne [ou soins de santé primaire $]^{18}$.

C'est dans ce contexte, que s'élabore le second Plan d'action en santé mentale 2005-2010: la force des liens ${ }^{20}$ [nommé PASM]. Celui-ci a pour objectif d'augmenter et de consolider l'offre de services en santé mentale en première ligne et dans la communauté. Les orientations du PASM auront pour effet de majorer de manière significative les services rendus à la clientèle présentant un problème modéré de santé mentale alors que jusque-là, on avait surtout investi les clientèles présentant un problème de santé mentale grave ${ }^{18}$.

Le "rétablissement" est vu comme le principe directeur du PASM tandis que l' "appropriation du pouvoir" tel que promu par le Plan d'action en santé mentale de $1998^{12}$ se voit remplacer par le "pouvoir d'agir"; certains considéreront que c'est une progression théorique et idéologique quant à la manière de percevoir les usagers ${ }^{21}$. Le "pouvoir d'agir" y est défini comme étant:

... la capacité des personnes souffrant d'un trouble mental de faire des choix et de participer activement aux décisions qui les concernent, et cela, en dépit de la présence chez elles de certains symptômes ou handicaps. La participation des utilisateurs et des proches aux exercices de planification des services de santé mentale découle de ce principe ${ }^{20}$.

Citant l'Organisation mondiale de la santé, le PASM insiste sur l'importance de la participation des usagers à la planification et à l'organisation des services et va même jusqu'à inscrire cette dernière, pour la toute première fois, à l'intérieur d'une mesure concrète:

Le MSSS, les agences de la santé et des services sociaux (ASSS) et les CSSS s'assureront d'obtenir la participation d'utilisateurs de services en santé mentale, de représentants des familles ou de proches dans l'exercice de planification et d'organisation de services qui les concernent ${ }^{20}$.

Le PASM incite également les partenaires du domaine de la santé mentale, mais extérieurs au réseau public de services, à favoriser .... la partici- pation des personnes utilisatrices de services au regard du mandat qu'ils assument, tout en offrant des conditions d'exercice adaptées aux capacités de ces dernières ${ }^{20}$. En somme, l'engagement de l'État québécois envers la participation des personnes utilisatrices de services de santé mentale, et la généralisation de ce processus à l'ensemble du Québec n'aura jamais été aussi clairement posé dans un document ministériel, aussi pleinement assumé.

Il semble que les acteurs du champ de la santé mentale aient reçu très positivement la mesure du PASM concernant la participation des usagers à la gouvernance des services de santé mentale au Québec. Celle-ci est vue comme un important levier pour multiplier les opportunités de participation des usagers et elle aurait contribué à étendre les lieux de participation des usagers aux trois paliers d'organisation des services: local, régional et national ${ }^{22}$.

Le PASM a aussi initié la décentralisation de la participation pour l'étaler jusque dans les régions rurales du Québec. Avant 2005, seulement deux régions du Québec possédaient une structure régionale d'organisation de la participation des usagers (structure organisée et permanente dont les objectifs sont habituellement et du moins théoriquement de se doter de modalité de représentation dans les différents lieux de participation). Désormais, 12 des 18 régions du Québec sont actives sur le plan de la participation des usagers et onze d'entre elles possèdent une structure formelle d'organisation de la participation des usagers (neuf ont été créés après l'adoption du PASM). Les régions qui possèdent une telle structure d'organisation de la participation ont un niveau d'engagement dans la gouvernance du système de santé beaucoup plus important que celles qui n'en ont pas. L'existence même de ces structures aurait en outre pour effet de rendre moins arbitraire l'engagement des usagers dans les activités de participation.

La plupart des structures d'organisation de la participation des usagers sont soutenues, dans l'exercice de leur mandat, par un organisme issu du milieu communautaire. Cette situation est contestée dans certains milieux d'usagers qui considèrent que cette responsabilité devrait plutôt être assumée par le réseau public de services puisque c'est lui qui commande et définit les activités auxquelles participent les usagers.

Les usagers impliqués dans les activités de participation bénéficient habituellement de mesures de soutien. Selon la forme prise par ces mesures (soutien technique et psychologique, formation à la participation, soutien financier...) 
elles sont l'initiative des structures d'organisations de la participation des usagers, des organismes communautaires et/ou du réseau public de services. Notons que dans certaines régions le financement s'avère très difficile à obtenir, demeure non suffisant ou encore est non récurrent.

Le rôle dont s'investissent les usagers dans les lieux de participation en est un de "porte-parole". Notons que plusieurs usagers disent ne pas trop savoir ce que l'on attend d'eux si ce n'est de parler de leur expérience de la maladie et des services de santé mentale. D'ailleurs, la plupart du temps les usagers prennent la parole et s'expriment avec le “je", postulant par le fait même que le "je" peut en effet avoir une valeur collective. Pour d'autres usagers, en contrepartie, on ne devrait jamais prendre la parole en son nom personnel dans les lieux où se pensent et s'organisent les services de santé mentale.

L'expérience de la maladie et des services est le socle à partir duquel les usagers participent. Par contre, les préoccupations qu'ils mettent de l'avant et défendent sont régulièrement autour d'autres enjeux fondamentaux (le logement, les droits et le travail). L'État de situation sur la participation des personnes utilisatrices suite au Plan d'action en santé mentale 2005-201022 suggèrent par ailleurs que les usagers ont une influence difficilement tangible sur les décisions qui se prennent. Ils contribueraient cependant à orienter les préoccupations qui se discutent.

\section{Conclusions}

À partir du Rapport Harnois ${ }^{1}$ jusqu'au dernier Plan d'action en santé mentale 2005-2010: La force des liens ${ }^{20}$, le MSSS est resté constant, quoiqu'à des degrés divers, dans sa volonté de faire participer les usagers à la gouvernance des services de santé mentale. Ce projet s'est toutefois passablement transformé renouvelant les manières avec lesquelles les usagers se sont inscrits dans la gouvernance du système de santé.

Le dernier Plan d'action en santé mentale 20052010: la force des liens a permis au MSSS de franchir une étape importante dans sa volonté de consolider la participation des usagers. L'invitation faite aux établissements du réseau public de services de "s'assurer de la participation des usagers" dans les exercices de planification et d'organisation des services est sans équivoque même si le projet porté par cette mesure est inachevé faute de repères concrets pour le réaliser. La notion de participation n’a pas été définie, le rôle attendu des personnes utilisatrices n'a pas été précisé, aucun budget n'a été prévu et ce, même si le ministère avait lui-même reconnu dans son Bilan d'implantation de la politique de santé mentale de $1997^{9}$ la "nécessité" d’un tel soutien. La question de la représentativité des personnes utilisatrices a été ignorée tandis que les établissements, pourtant tenus de "s'assurer de la participation des usagers", n'ont été soumis à aucune reddition de compte.

L'engagement réel et contributif des usagers aux exercices de planification et d'organisation des services de santé mentale ne peut tenir qu'à des principes inscrits dans des documents ministériels. Il faut des cibles à atteindre, des processus de reddition de compte et des responsabilités clairement énoncées. La question du financement est aussi centrale, car lorsque le financement affecté à ces structures cesse, les activités de participation cessent également.

Enfin, est-ce que les aspirations, les préoccupations et les changements souhaités par les usagers s'inscrivent dans une simple volonté de continuité ou dans une volonté de renouvellement de la démocratie? On peut s'interroger sur le projet porté par les usagers inscrits dans la gouvernance du système de santé, car, pour le moment, leur participation semble s'appuyer sur un consensus dont personne n'est en mesure de préciser les termes. Il reste encore à répondre à la question: pourquoi participer?

\section{Colaborateurs}

M. Clément, LR del Barrio, J Gagné et A Lévesque ont participé également à toutes les étapes d'élaboration de cet article.

\section{Remerciements}

Les auteurs tiennent à remercier les membres du comité citoyen de l'ARUCI-SMQ pour leu précieux commentaires à l'étape de la construction de ce texte ainsi que Thaïs Mikie de de Carvalho Otanari et Sabrina Steffanello pour leur soutien à la version portugaise de cet article. 


\section{Références}

1. Canada. Gouvernement du Québec. Ministère de la Santé et des Services Sociaux (MSSS). Politique de santé mentale. Québec: MSSS; 1989.

2. Canada. Gouvernement du Québec. Comissaire à la Santé et au Bien-être (CSBE). Participation et consultation des citoyennes et citoyens en matière de santé et de bien-être. Québec: CSBE; 2004. p. 62.

3. Thibault A., Lequin M., Tremblay M. Cadre de référence de la participation publique (démocratique, utile et crédible) Proposé pour avis aux citoyens actifs du Québec, par le groupe de travail sur la qualité de la participation publique formé à la suite du Forum sur le développement social. Québec: CSBE; 2000.

4. Tremblay M. Droits, santé et participation démocratique. Participation démocratique et publique. 2004; 17(1):41-49.

5. Canada. Gouvernement du Québec. Ministère de la Santé et des Services Sociaux (MSSS). Pour un partenariat élargi. Projet de politique de santé mentale pour le Québec. Québec: MSSS; 1987. p. 185.

6. Fleury MJ, Grenier G. Historique et enjeux du système de santé mentale québécois. Rupture 2004; 10(1):31-38.

7. Canada. Gouvernement du Québec. Comité de santé mentale du Québec (CSMQ). Recommandations pour développer et enrichir La Politique de santé mentale. Québec: CSMQ, Les publications du Québec; 1994. p. 60.

8. Canada. Gouvernement du Québec. Comité de santé mentale du Québec (CSMQ). Vers une politique de santé mentale de 2ième génération: rapport d'activités pour les années 1989 à 1995. Québec: CSMQ; 1995. p. 101.

9. Canada. Gouvernement du Québec. Ministère de la Santé et des Services Sociaux (MSSS). Bilan d'implantation de la politique de santé mentale. Québec: MSSS; 1997. p. 159.

10. Canada. Gouvernement du Québec. Vérificateur général du Québec (VGQ). Rapport du Vérificateur général à l'Assemblée nationale pour l'année 1995 1996 Tome II - Action du gouvernement relativement aux organismes communautaires de la santé et des services sociaux et à la santé mentale. Québec: VGQ; 1996. p. 25.

11. Canada. Gouvernement du Québec. Ministère de la Santé et des Services Sociaux (MSSS). Orientations pour la transformation des services de santé mentale. Québec: MSSS; 1997. p. 51.

12. Canada. Gouvernement du Québec. Ministère de la Santé et des Services Sociaux (MSSS). Plan d'action pour la transformation des services en santé mentale. Québec: MSSS; 1998. p. 41.

13. Rodriguez L, Guay L, Bourgeois L, Landry Y, Pinard JL. Repenser la qualité des services dans la communauté. Changer de perspective. Cadre de référence pour la qualité du point de vue des personnes utilisatrices des services en santé mentale. In: Comité de la santé mentale du Québec. Montréal: Presses de l’Université du Québec; 2006.
14. Canada. Gouvernement du Québec. Ministère de la Santé et des Services Sociaux (MSSS). Transformation des services de santé mentale. État d'avancement du plan d'action de décembre 1998. Québec: MSSS; 1999. p. 50

15. Canada. Gouvernement du Québec. Ministère de la Santé et des Services Sociaux (MSSS). Accentuer la transformation des services de santé mentale. Cibles prioritaires adoptées au Forum sur la santé mentale de septembre 2000. Québec: MSSS; 2001. p. 52.

16. Godrie B. Forum des personnes utilisatrices. Participation à la planification et à l'organisation des services de santé mentale. Compte rendu du Forum tenu dans le cadre des 8ièmes Journées bi-annuelles de santé mentale au Québec le 11 mai 2010, Hôtel Delta Centre-Ville, Montréal. Montréal; 2010. Document non publié.

17. Canada. Gouvernement du Québec. Vérificateur général du Québec (VGQ). Rapport annuel du vérificateur du Québec à l'Assemblée Nationale pour l'année 2002-2003. Tome II. Québec: VGQ; 2003.

18. Canada. Gouvernement du Québec. Comissaire à la Santé et au Bien-être (CSBE). État de situation sur la santé mentale au Québec et réponse du système de santé et de services sociaux. Québec: CSBE; 2012. p. 232.

19. Canada. Gouvernement du Québec. Ministère de la Santé et des Services Sociaux (MSSS). Projet de loi 83: Loi modifiant la Loi sur les services de santé et les services sociaux et d'autres dispositions législatives. Québec: MSSS; 2005.

20. Canada. Gouvernement du Québec. Ministère de la Santé et des Services Sociaux (MSSS). Plan d'action en santé mentale. La force des liens, 2005-2010. Québec: MSSS; 2005.

21. Bouchard R. Présentation de Réjeanne Bouchard lors de la journée d'étude en santé mentale du 24 avril 2009. Revue Santé mentale au Québec. [serial on the Internet]. [cited 2013 Ago 2]. Available from: http://www.santementaleauquebec.ca/pdf/ bouchard.pdf

22. Clément M, Rodriguez del Barrio L, Gagné J, Lévesque A, Vallée C. État de situation sur la participation des personnes utilisatrices suite au Plan d'action en santé mentale 2005-2010. Rapport de recherche; ARUCI-SMC; 2012.

Artigo apresentado em 29/04/2013

Aprovado em 13/05/2013

Versão final apresentada em 05/06/2013 